\title{
Designing and testing lightweight shoulder prostheses with hybrid actuators for movements involved in typical activities of daily living and impact absorption
}

\author{
This article was published in the following Dove Press journal: \\ Medical Devices: Evidence and Research \\ 6 July 2015 \\ Number of times this article has been viewed
}

\author{
Masashi Sekine ${ }^{1,2}$ \\ Kahori Kita' \\ Wenwei Yu' \\ 'Center for Frontier Medical \\ Engineering, ${ }^{2} \mathrm{Graduate}$ School \\ of Engineering, Chiba University, \\ Chiba, Japan
}

Correspondence: Masashi Sekine Center for Frontier Medical Engineering, Chiba University, I-33 Yayoicho, Inageku, Chiba 263-8522, Japan

Tel $+8 I 432903 I I 2$

Fax +8I43290 3III2

Email sekine@office.chiba-u.jp

\begin{abstract}
Unlike forearm amputees, transhumeral amputees have residual stumps that are too small to provide a sufficient range of operation for their prosthetic parts to perform usual activities of daily living. Furthermore, it is difficult for small residual stumps to provide sufficient impact absorption for safe manipulation in daily living, as intact arms do. Therefore, substitution of upper limb function in transhumeral amputees requires a sufficient range of motion and sufficient viscoelasticity for shoulder prostheses under critical weight and dimension constraints. We propose the use of two different types of actuators, ie, pneumatic elastic actuators (PEAs) and servo motors. PEAs offer high power-to-weight performance and have intrinsic viscoelasticity in comparison with motors or standard industrial pneumatic cylinder actuators. However, the usefulness of PEAs in large working spaces is limited because of their short strokes. Servo motors, in contrast, can be used to achieve large ranges of motion. In this study, the relationship between the force and stroke of PEAs was investigated. The impact absorption of both types of actuators was measured using a single degree-of-freedom prototype to evaluate actuator compliance for safety purposes. Based on the fundamental properties of the actuators identified, a four degree-of-freedom robotic arm is proposed for prosthetic use. The configuration of the actuators and functional parts was designed to achieve a specified range of motion and torque calculated from the results of a simulation of typical movements performed in usual activities of daily living. Our experimental results showed that the requirements for the shoulder prostheses could be satisfied.
\end{abstract}

Keywords: shoulder prosthesis, hybrid actuation, pneumatic elastic actuator, antagonistic mechanism, compliance

\section{Introduction}

A prosthesis can perform sensorimotor functions for an amputee. If every such function could be performed by a prosthesis, it would approach the usefulness of a healthy human limb, but this would increase the number of parts and devices required, which would increase the cost, weight, and difficulty of maintenance. Therefore, it is more realistic to provide a prosthesis that performs fewer functions than a real human limb. As such, prostheses including a terminal device and a control system should be developed with a focus on priorities most suitable for users, considering the usage conditions. ${ }^{1}$ A previous study ${ }^{1}$ focused on issues concerning the usability of myoelectric controls and upper limb prostheses. For example, because unilateral arm amputees are considered able to perform many activities of daily living (ADLs) with their intact 
arm, it is reasonable to develop prostheses with functions that support bimanual coordination motions, except for one-handed ADLs. On the one hand, the forms of prostheses vary depending on the amputated limb. Research examples of prosthetic hands ${ }^{2-5}$ and other types ${ }^{6-11}$ of prostheses have been reported. A shoulder prosthesis is difficult to develop because of the larger dimensions required. Unlike forearm amputees, transhumeral amputees have residual stumps that are too small to provide a sufficient range of operation for their prosthetic parts to perform usual ADLs. Further, it is difficult for small residual stumps to provide sufficient impact absorption for safe manipulation in ADLs, as intact arms do. Therefore, it is important that a shoulder prosthesis has a sufficient range of motion (ROM) and sufficient compliance for the required motions of the user. These requirements inevitably require a shoulder prosthesis to have more parts and actuators and to weigh more than a hand or elbow prosthesis. However, lightness is one of the most important features of a prosthesis. For example, it was reported that $58 \%$ of respondents to a questionnaire given to hemilateral forearm amputees about requirements and desired improvements on the hardware, services, and schemes for use of an electric upper limb prosthesis hoped for further reduction of the weight and price (multiple answers allowed) than functionality, appearance, or a public prosthetic supply system in Japan; moreover, almost all the respondents answered that the desirable weight should be less than $0.9 \mathrm{~kg}{ }^{12}$ The development of a shoulder prosthesis is made difficult by the trade-off between functionality and lightness.

In other studies, higher degree-of-freedom (DOF) shoulder-arm prostheses driven by motors have been developed. ${ }^{13-15}$ In their paper, Fukaya et $\mathrm{al}^{15}$ presented an effort to develop a 13-DOF shoulder prosthesis the whole weight of which was $3.885 \mathrm{~kg}$, with a spherical ultrasonic motor. These prostheses are multifunctional, especially one that is an arm ${ }^{14}$ controlled using targeted muscle reinnervation, which is considered state-of-the-art technology. However, these prostheses are not light enough; each has a total weight of approximately 4-6 kg. Amputees prefer a prosthetic arm that weighs less. ${ }^{12,16}$ For example, Yokoyama et $\mathrm{al}^{16}$ reported that a high-level upper limb amputee felt the prosthetic arm with a myoelectric hand of $1.7 \mathrm{~kg}$ to be heavy when wearing it. Another development in prostheses is the use of motors, which have become common and can potentially provide both a wide ROM and a stable output force. However, the motors that are usually used are not lightweight in comparison with their payload. Moreover, such motors lack back-drivability, which is important for safe use because of the strong torques and multistage reduction gear. This means that a prosthetic arm with insufficient compliance, for example, could cause unexpected injuries because the arm would not be able to absorb the impact of contacts or collisions but rather would transmit the impact force directly to the socket, ie, the user's body. Although a motor can possess pseudocompliance through appropriate controls, the time delay involved in absorbing an impact remains an issue. Because the time span of a collision impact's peak value is very short, it would be ideal to be able to prevent any time delay. For example, development of robotic hands, arms, and medical devices with non-control-based and intrinsic flexibility that appear to be adaptable to prostheses has been proposed. ${ }^{17-20}$

Pneumatic elastic actuators (PEAs) have intrinsic flexibility. These are well suited to safe use in prostheses because they are both lightweight and soft, as a result of the viscoelasticity of air and rubber, and thus provide impact absorption without a time delay. PEAs do not need any additional passive elements for safety purposes. The use of PEAs could significantly reduce the weight and volume of a prosthesis. A smaller volume improves the appearance of a prosthesis, which is a key design consideration. Moreover, according to Plattenburg et al, PEAs offer high power-toweight performance in comparison with motors or standard industrial pneumatic cylinder actuators. ${ }^{3,21,22}$ Given the necessary characteristic features of a prosthesis, incorporation of PEAs could be useful in minimizing weight while providing a high output force and sufficient compliance for impact absorption. However, it is difficult to achieve a sufficient ROM with a PEA because of the short stroke (shrinkage length) and antagonistic mechanism (AM) required in a joint composed of PEAs. In addition, the output force is not necessarily stable with respect to the stroke. A motor offers advantages over PEAs in terms of the larger ROM possible, which contributes to a larger working space for the prosthesis and a stable output force (torque). Therefore, it is possible to use PEAs for designing ideal shoulder prostheses in combination with a motor to exploit the advantages of each and compensate for their respective shortcomings.

In this study, a hybrid lightweight shoulder prosthesis that uses the two aforementioned types of actuators was designed. In the design of the shoulder prosthesis, the layout of the two types of actuators was optimized to produce a lightweight device with sufficient compliance, ROM, and output force. The properties of the two types of actuators were first compared. The relationship between the force and shrinkage length of a PEA was investigated empirically. 
The impact absorption characteristics of both types of actuators were measured using a simplified 1-DOF arm and prototype measuring system.

In this study, we developed a 4-DOF linked arm model for the shoulder prosthesis and simulated the required rotational angle and torque of the joints by making the arm model track a certain pathway considered to represent a typical ADL motion. The layout of the two types of actuators in the joints of the model was determined from the results of the simulation. We confirmed that the PEA and the joint combined with it in the 1-DOF arm had better impact absorption properties than the motor and that the 4-DOF linked arm model was able to track the intended pathway and enhance the impact absorption properties of the prototype. The layout of the hybrid actuators in the joints was based on the results of the simulation and experiments.

A design procedure that considers the working space, safety, lightness, and torque of a shoulder prosthesis is described in this paper, and the design of a hybrid shoulder prosthesis developed using the procedure is presented. Basically, the ROM, compliance (for impact absorption), arm lightness, output torque, speed and stiffness of hand and joint, energy consumption, volume, and weight of a wearable power supply are considered as requirements for development of prostheses. These factors are closely related. In this study, the impact absorption, arm weight, and ROM requirements were to be satisfied through hybridization of two different types of actuators; meanwhile, the torque and volume requirements were also met, considering typical ADL movements, so this study contributes to the development of robotic arms for prosthetic uses. Other requirements are to be addressed in a subsequent project. The definitions of symbols used frequently in this paper are shown in Table 1.

\section{Materials and methods Characteristics of PEA}

\section{Compliance (impact absorption)}

In this study, the PEA (PM-10P, SQUSE Inc.) shown in Figure $1 \mathrm{~A}$ was employed. This is lightweight $(0.003 \mathrm{~kg})$ and has

Table I Definition of symbols used frequently in this paper

\begin{tabular}{ll}
\hline$F_{t}$ & Traction force \\
$x_{s l}$ & Shrinkage length \\
$T$ & Required torque \\
$T_{A M}$ & Torque in antagonistic mechanism \\
$\mathrm{J}$ & Joint \\
$\phi$ & Joint angle \\
$\mathrm{L}$ & Link \\
$I$ & Length of link \\
\hline
\end{tabular}

a high output force (maximum pulling force $100 \mathrm{~N}$ ) and average shrinkage ratio of $30 \%$ per piece. These data are shown in its product catalog. ${ }^{23}$ The maximum power-to-mass ratio is 33.3 $\mathrm{kN} / \mathrm{kg}$. This actuator makes it possible to generate a force only in flexion. Therefore, a unidirectional translational motion of the PEA can be converted into a bidirectional rotational motion using an AM, as shown in Figure 1B, and the actuator can be used in the joint of the prosthesis. In addition, the actuator has compliance in both the long axial direction and the direction around the long axis (perpendicular to the external surface of the actuator), as shown in Figure 1C and 1D. Therefore, placing PEAs around the base frame, mimicking a mechanism of human muscles and bony framework, as shown in Figure 1B, gives rise to the possibility of absorbing impact force to a user's body when the prosthetic arm collides with external objects. These characteristics were confirmed in the experiments described in a later section. Use of PEA is considered to contribute to compliance, ie, safe use of a prosthesis.

\section{Spring constant}

It is necessary to determine the relationship between the traction force $F_{t}$ and the shrinkage length $x_{s l}$ of the PEA, ie, the spring constant $k$ required to calculate the required joint torque and rotational angle of the AM in the shoulder prosthesis. The equation was assumed to be a linear function of the following form, where the constant is denoted by $c$.

$$
F_{t}=k x_{s l}+c
$$

The method used is shown in Figure 2. Three pieces of PEAs were connected serially for averaging purposes. The upper end of the series of PEAs was fixed to the test frame. Weights from $9.8 \mathrm{~N}$ to $98 \mathrm{~N}$ (10 kg; acceleration due to gravity $\left.\mathrm{g}=9.8 \mathrm{~m} / \mathrm{sec}^{2}\right)$ were added in increments of $9.8 \mathrm{~N}(1 \mathrm{~kg})$ to the lower end of the series of PEAs, and air pressure was conveyed to the actuators at a maximum value of $0.2 \mathrm{MPa}$. The change in length $x_{s l}$ with respect to the unloaded natural length of each PEA piece was measured under each weight. Because PEAs exhibit characteristics of hysteresis, this was also measured.

\section{Comparison between PEA and motor}

An experiment was conducted to assess the effect of compliance in embedding a PEA in a prosthesis. The compliance was evaluated in terms of the level of absorption of an impact force from an external collision. This was measured in both the long axial direction and the direction around the long axis of the PEA, as shown in Figure 1C. For the long axial direction, an AM consisting of PEAs was developed, and the 
A

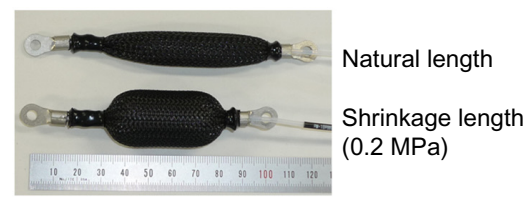

Pneumatic elastic actuator

C

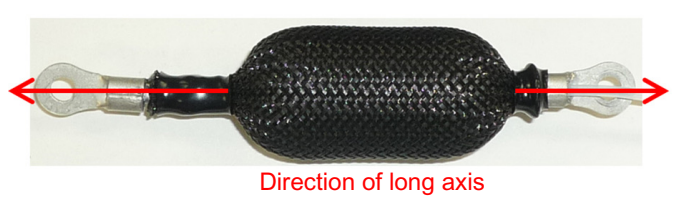

Compliance in two directions
B

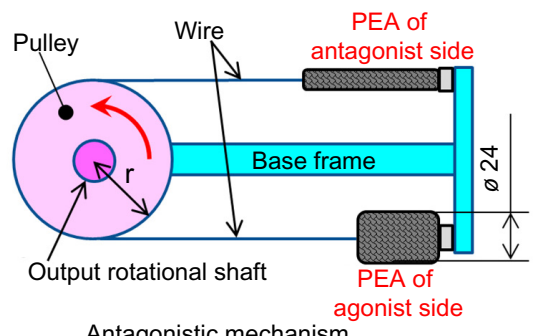

Antagonistic mechanism

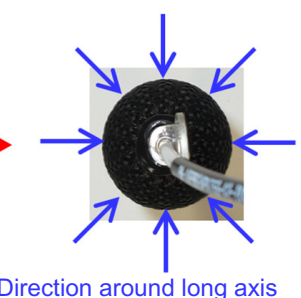

D

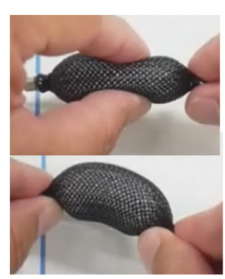

Responses to pinching and bending

Figure I Characteristics of PEA.

Notes: (A) PEA; (B) AM with PEA; (C) compliance in two directions; (D) responses to pinching and bending.

Abbreviations: AM, antagonistic mechanism; PEA, pneumatic elastic actuator.

level of absorption of the impact force on the output rotational shaft of the AM was compared with that of a motor.

\section{Direction around long axis}

The method used to test the impact absorption of a PEA in the direction around its long axis is shown in Figure 3. First, the PEA was tested using a force gauge (FGP-50, ${ }^{24} \mathrm{Nidec}-\mathrm{Shimpo}$ Corporation, $\pm 500 \mathrm{~N}$ ), with the external surface of the PEA placed on the measurement surface of the force gauge, as shown in Figure 3A. A weight of $0.98 \mathrm{~N}$ was dropped from a height of $30 \mathrm{~mm}$ and collided with the surface of the PEA

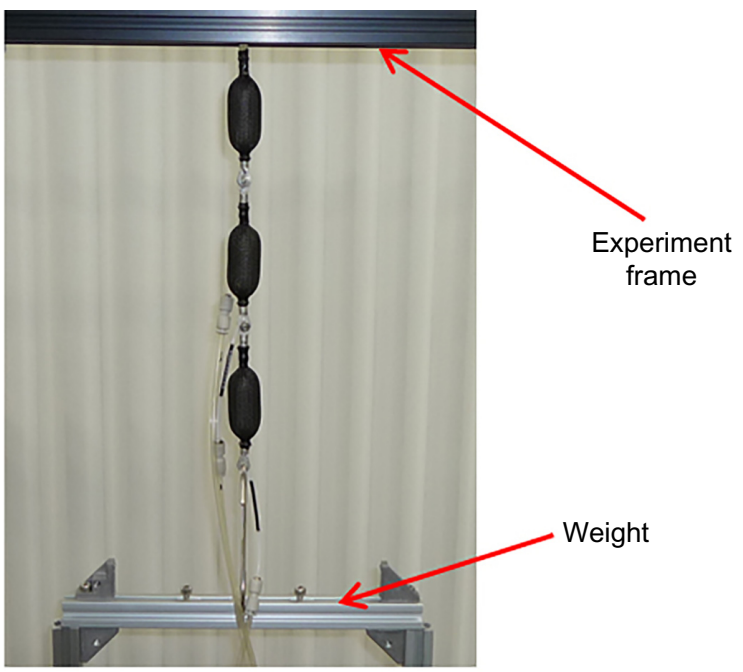

Activated (0.2 MPa)

Figure 2 Measurement of spring constant.

Note: A weight of $9.8 \mathrm{~N}$ is applied in the figure.

Abbreviation: PEA, pneumatic elastic actuator. (in the direction around long axis), and the impact force was measured. A weight of $0.98 \mathrm{~N}$ was also dropped from a height of $30 \mathrm{~mm}$ and collided with the measurement surface directly, without the PEA in place. In this way, the effect of the PEA in the impact force absorption was confirmed. This test was performed using air pressures of $0.05,0.1$, and $0.2 \mathrm{MPa}$. The weight was connected to a slider and traveled along the slider's rail as it fell. For the cases of a 0.2 MPa air pressure in the PEA and direct impact, weights from $0.98 \mathrm{~N}$ to $9.8 \mathrm{~N}(1 \mathrm{~kg})$ were applied in increments of $0.98 \mathrm{~N}$, and the trend of response with increasing load was examined. As described in detail later in this paper, if a significant difference exists in impact absorption with and without the PEA, the need to place PEAs around the base frame (link), as shown in Figure 1B, is confirmed.

\section{Direction of long axis}

The method used to measure the impact absorption of the PEA in the direction of the long axis is illustrated in Figure 4. The 1-DOF arm was prepared as shown in Figure 4A. The arm was linked to the sliding base with a rotational shaft and ball bearings. The sliding base was placed on the measurement surface of the force gauge. The sliding base was not fixed to the measurement surface but rather positioned on the surface in a way that allowed it to slide up and down on the slide rail. The output shaft of the AM with the PEAs (shown in Figure 4B and D) or the motor (RS405CB,${ }^{25}$ Futaba Corporation, $0.067 \mathrm{~kg}, 4.7 \mathrm{~N} \cdot \mathrm{m}(48.0 \mathrm{~kg} \cdot \mathrm{cm}$; shown in Figure 4C and $\mathrm{E}$ ) was connected to the arm's rotational shaft. A weight of $0.98 \mathrm{~N}$ was dropped from a height of $30 \mathrm{~mm}$ and collided with the arm, as shown in Figure 4, and the impact forces 


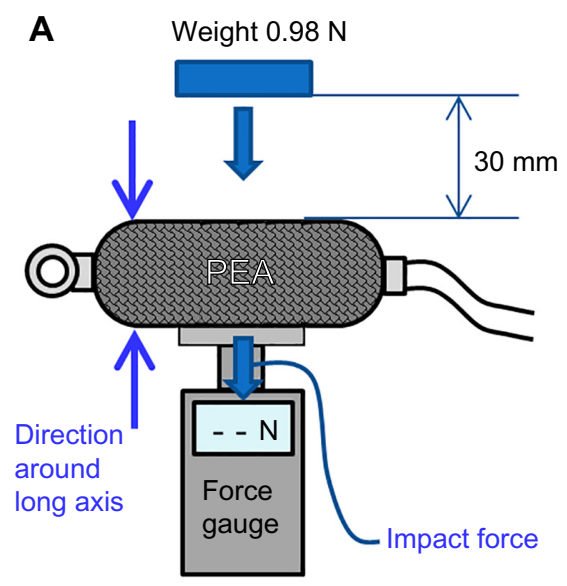

Impact force measured by force gauge with absorption by PEA
B

Weight $0.98 \mathrm{~N}$

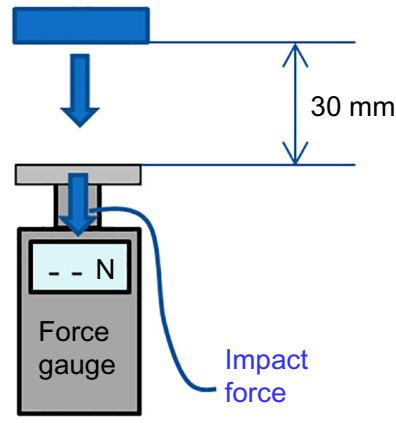

Direct impact force Measured by force gauge

Figure 3 Measuring compliance in direction around long axis.

Notes: (A) Weight collides to PEA placed on the measurement surface of the force gauge; (B) weight collides to the measurement surface of the force gauge directly. Abbreviation: PEA, pneumatic elastic actuator.

were measured. The degrees of impact absorption along the rotational axis, ie, the joints consisting of the motor and the $\mathrm{AM}$, were compared, ie, the compliance in the direction of the long axis of the PEAs making up the AM was compared with that of the motor. As described in the previous section, air pressures of $0.05,0.1$, and $0.2 \mathrm{MPa}$ were used. The measuring system is shown in Figure 4D and E.

\section{Combination of two directions}

Figure 4F and G show the PEA in place for testing in the configurations shown in Figure 4D and E, respectively, ie, the combination of the impact absorption of the joint provided by the PEA and motor with the absorption of the PEA in the direction around its long axis was confirmed. The weight on the PEA and the air pressure of the PEA were $0.98 \mathrm{~N}$ and $0.2 \mathrm{MPa}$, respectively.

\section{Modeling Configuration of 4-DOF link arm model}

In this study, a two-link arm model with four DOFs was developed, as shown in Figure 5. The two links were the $\mathrm{L}_{\mathrm{U}}$ of the upper arm and the $\mathrm{L}_{\mathrm{FH}}$ of the forearm with the hand. Two DOFs were established in the shoulder (two joints, $\mathrm{J}_{1}$ and $\mathrm{J}_{2}$ ) and two in the elbow (two joints, $\mathrm{J}_{3}$ and $\mathrm{J}_{4}$ ). Although 1-DOF grasping capability is normally established for the hand, this was not a factor considered in the simulation conducted to
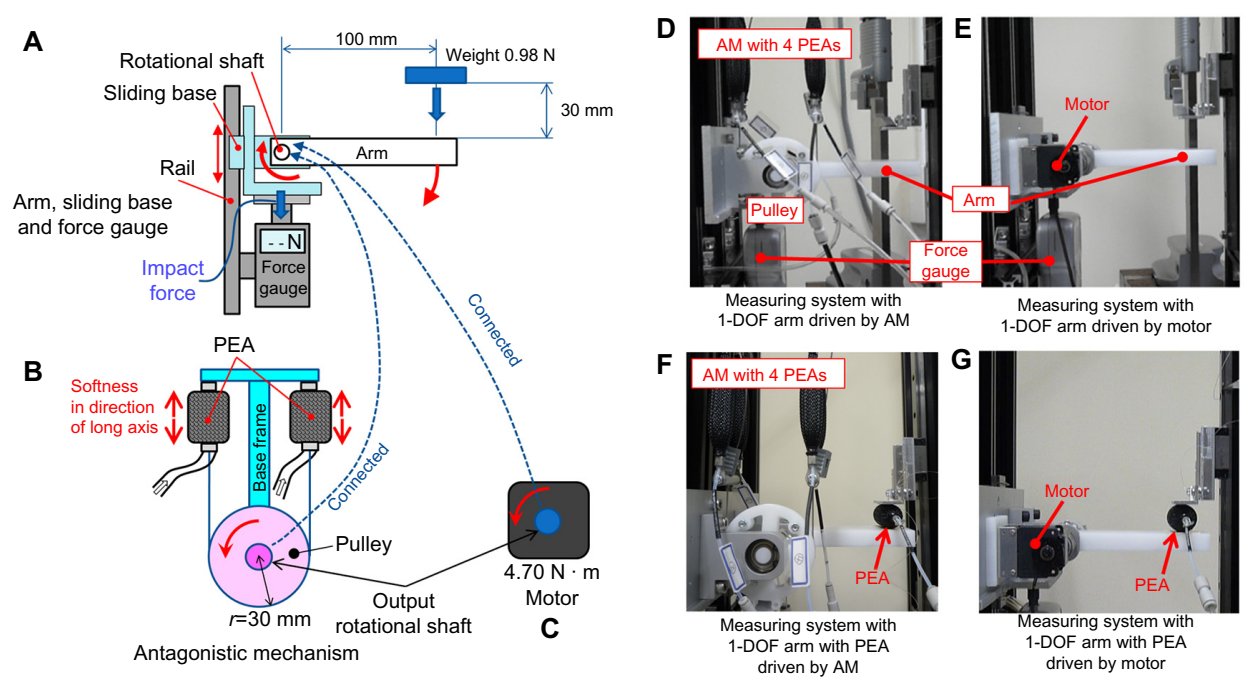

Figure 4 Measuring compliance of PEA in direction of long axis with and without PEA in direction around long axis.

Notes: (A) Measuring system; (B) AM with PEA; (C) servo motor; (D) measuring system driven by AM; (E) measuring system driven by motor; (F) measuring system driven by AM with PEA on I-DOF arm; (G) measuring system driven by motor with PEA on I-DOF arm.

Abbreviations: AM, antagonistic mechanism; PEA, pneumatic elastic actuator; I-DOF, single degree-of-freedom. 


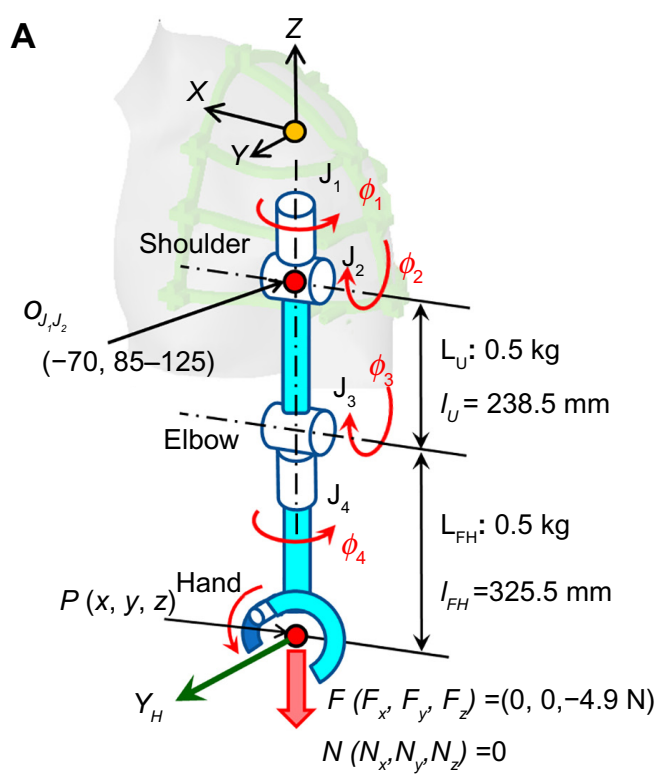

4-DOF arm link model

\section{B}

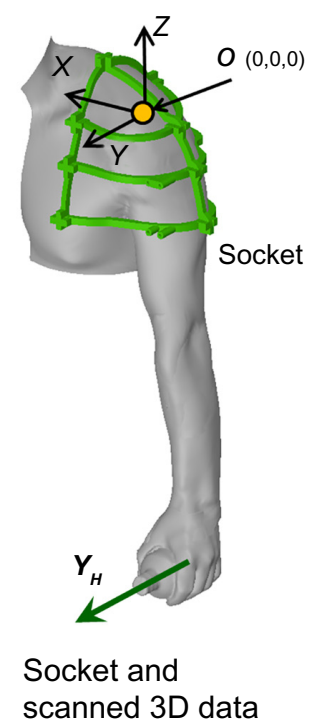

Figure 5 Arm link model of shoulder prosthesis.

Notes: (A) 4-DOF arm link model; (B) scanned 3D data of socket and subject's body surface. See Table I for definitions of symbols. Abbreviations: 3D, three-dimensional; 4-DOF, four degrees-of-freedom.

optimize the actuator layout in this study. This weight and geometry (described later) were used just to create a complete shoulder prosthesis. Hand-to-mouth and hand-to-face movements, such as those involved in eating and hygiene activities, are important movements required for a prosthesis in daily living. For such movements, a larger angle of flexion of the elbow joint is assumed to be required, and this was confirmed in the simulation. In this study, we assumed that the prosthesis being developed would not be adapted for amputees immediately but rather for healthy subjects first to validate the design and its performance to some extent. Therefore, body surface data for the shoulder to the hand of one healthy subject (170.5 cm tall) were scanned and acquired using a three-dimensional scanner (Artec MHT), and a socket fitting the shoulder was designed assuming the prosthesis would be worn as shown in Figure 5B. Movement of the prosthetic arm was simulated under these conditions. The link length was based on the arm length determined from the scanned data, as shown in Figure 5A. The weight of the link model was based on that of a similar prototype arm that we developed in previous research ${ }^{26,27}$ and the target weight was $1.0 \mathrm{~kg}$. The load at the hand was assumed to be $4.9 \mathrm{~N}$, considering a realization of typical ADL hand-to-mouth movements such as drinking a glass of water.

\section{Calculating of joint angle and torque}

As Figure 5B shows, the origin of the global coordinate system $O-X Y Z$ was placed at the left acromion. The intersection

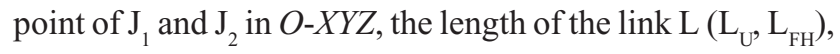
the joint angle $\mathrm{J}\left(\mathrm{J}_{1}, \mathrm{~J}_{2}, \mathrm{~J}_{3}, \mathrm{~J}_{4}\right)$, and the coordinates of the hand position (ie, the end tip of $\mathrm{L}_{\mathrm{FH}}$ ) were defined as $O_{J 1 / 2}$, $l\left(l_{U} l_{F H}\right), \phi\left(\phi_{1}, \phi_{2}, \phi_{3}, \phi_{4}\right)$, and $P(x, y, z)$, respectively. The following equations were used to simulate the required angle $\phi$ and torque $T\left(T_{1}, T_{2}, T_{3}, T_{4}\right)$ of each joint in the link model. The relationship between $\phi, l$, and $P$ was calculated using equation (2). Equation (3) is the dynamic equation for the $T$ of J, the external force $F$ and $\phi$. The $\phi$ was calculated by substituting the hand pathway into equation (2) using a numerical solution, and $T$ was derived by substituting $\phi$ into equation (3).

$$
\begin{gathered}
P=O_{J 1,2}+f(l, \phi) \\
T=H(\phi) \ddot{\phi}+C(\phi, \dot{\phi})+G_{f}(\phi) g+J_{f}^{T}(F, N)
\end{gathered}
$$

$\mathrm{H}(\phi)$ : inertia term, $\mathrm{C}(\phi, \dot{\phi})$ : Coliolis term, $\mathrm{G}_{f}(\phi)$ : gravity term, $J_{f}^{T}(F, N)$ : external force term including transposed Jacobian matrix, $(F, N)$ : external force $F\left(F_{x}, F_{y}, F_{z}\right)$ and moment $N\left(N_{x}, N_{y}, N_{z}\right)$ acting on $P^{28}$

For the case of the AM for the joint J, shown in Figure 1B, $F_{t}$, and $x_{s l}$ of the PEA were derived by substituting the angle $\phi$ (in degrees) into equation (4) below using equation (1). Here, $r$ is the radius of the pulley, ie, the moment arm, as shown in Figure 1B. The output torque of the AM, $T_{A M}\left(T_{A M 1}, T_{A M 2}\right.$, $T_{A M 3}, T_{A M 4}$ ), was calculated using equation (5).

$$
x_{s l}=2 \pi r \phi / 360
$$




$$
T_{A M}=F_{t} r=k x_{s l} r+c r
$$

\section{Method for determining PEA configuration in $A M$ and layout of PEA and motor in each joint}

In applying equation (5), one PEA piece on the agonistic side and one on the antagonistic side are considered, ie, two pieces in total, as shown in Figure 1B. It is possible that two PEA pieces are unable to provide the required torque $T$ and joint angle $\phi$, depending on the hand pathway superimposed on the link arm. In this case, multiple connected PEAs are required. The PEAs can be connected serially or in parallel, or in a combination of the two, in whatever manner the greatest output torque and rotational angle of the AM can be efficiently provided. The use of multiple PEAs is illustrated in Figure 6. Let the number of serial and parallel connections in the joint $\mathrm{J}$ be $n_{s}\left(n_{s 1}, n_{s 2}, n_{s 3}, n_{s 4}\right)$ and $n_{p}\left(n_{p 1}, n_{p 2}, n_{p 3}, n_{p 4}\right)$, respectively. Equation (5) can be transformed into equation (6) to reflect this situation. Finally, the selection between using PEAs or using a motor for each $\mathrm{J}$ is made on the basis of the calculated value of the total weight for the smallest number of PEAs, pulleys, and accessories to provide the required $T$ and $\phi$ for each J, given the experimental results described in the section on direction around long axis. Although it is difficult to make a decision based on weight without consideration of the degree of impact absorption, in this study a servo motor was selected as an actuator in a joint to substitute an $\mathrm{AM}$ when the AM weighs more than two motors, ie, 0.134 $\mathrm{kg}$ (the weight of one motor is $0.067 \mathrm{~kg}$ ). This weight-based decision criterion resulted from comprehensively considering the volume, weight, ROM, and appearance of prostheses. Although other small parts such as screws are necessary for the $\mathrm{AM}$, the motor requires similar parts, so these were not considered in the comparison. The results presented in the previous section confirm the advantage of the PEA in terms
A



B

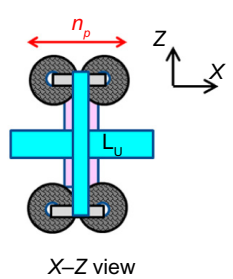

Figure 6 Case of multiple actuators in antagonistic mechanism $\left[2\left(n_{s} \times n_{p}\right)=8\right.$ pieces of actuator].

Notes: (A) Y-Z view of AM; (B) $X-Z$ view of AM. See Table I for definitions of symbols.

Abbreviation: AM, antagonistic mechanism. of safety. The significant difference in impact absorption capacity of the PEA in the direction around its long axis, compared with that of direct impact (without a PEA), is confirmed by the experimental results described in the previous section. Therefore, the requirement for PEAs around the link (base frame), as shown in Figure 6, was identified as a basic design principle. However, in the case of $n_{s}>2$, because the total length of PEAs in the direction of the long axis is greater than the link length $l\left(l_{U} l_{F H}\right)$, PEAs are not placed on the link $L$ but rather on a backpack and actuated remotely using flexible Bowden cables. This backpack system was employed in our previous study. ${ }^{26,27} \mathrm{~A}$ bevel-gear drive box was employed when the base frame shown in Figure 1B was assumed to be the $\mathrm{L}_{\mathrm{FH}}$ in Figure $5 \mathrm{~A}$, because the axis direction between the pulley and the joint rotation differs from $90^{\circ}$ in the relationship between $\mathrm{J}_{4}$ and $\mathrm{L}_{\mathrm{FH}}$. The box's weight must be determined. The radius $r$ of the pulley in the AM in each $\mathrm{J}$ of the 4-DOF arm was set to $15 \mathrm{~mm}$ because the diameter of the PEA when compressed fully was approximately $24 \mathrm{~mm}$, and the contraction of an agonistic PEA would interfere with the antagonistic PEA in the AM, when $r$ was less than $12 \mathrm{~mm}$, as shown in Figure 1B.

$$
T_{A M}=F_{t} r=n_{p}\left(k x_{s l} / n_{s}+c\right) r
$$

\section{Confirmation of layout by CAD model}

The layout of the actuators and the shape of the links were designed and validated using a computer-aided design (CAD) software model. The output impact force on the shoulder with respect to the input impact force on the link $\mathrm{L}_{U}$ in the horizontal $(X)$ and vertical $(Z)$ directions in the case of the whole arm extending in the $Y$ direction $\left(\phi_{2}=90^{\circ}\right)$ was estimated based on the results described in the section on combination of two directions. For example, when the base frame and the AM were assumed to be the $\mathrm{L}_{\mathrm{U}}$ and joint $\mathrm{J}_{3}$ in Figures 6 and 7, respectively, even for a fixed number of PEAs, the impact on the shoulder varies based on the layout of the PEAs. As shown in Figure 7, assuming $\mathrm{L}_{\mathrm{U}}$ and $\mathrm{L}_{\mathrm{FH}}$ together are able to produce an effect of the PEAs in the direction around the long axis in response to an external impact force by overlaying the PEAs with the cover, the effects of impact absorption can be expected only in the vertical direction for the case shown in Figure 7A and in both the horizontal and vertical directions for the case shown in Figure 7B.

\section{Hand pathway superimposed on link arm}

The pathway of the hand (the end tip of $\mathrm{L}_{\mathrm{FH}}$ ) that was superimposed on the link arm is shown in Figure 8. The pathway 
A

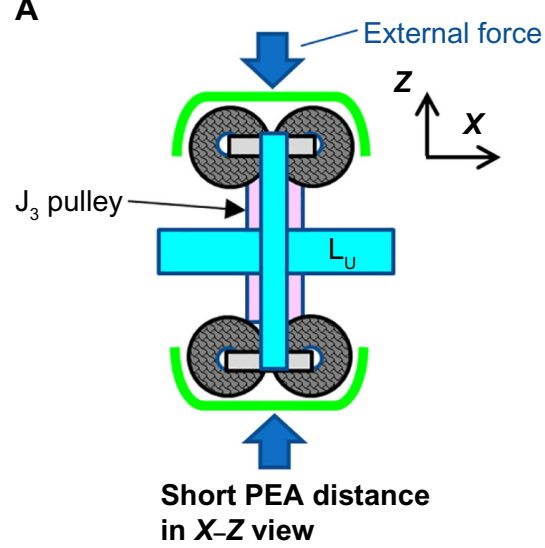

B

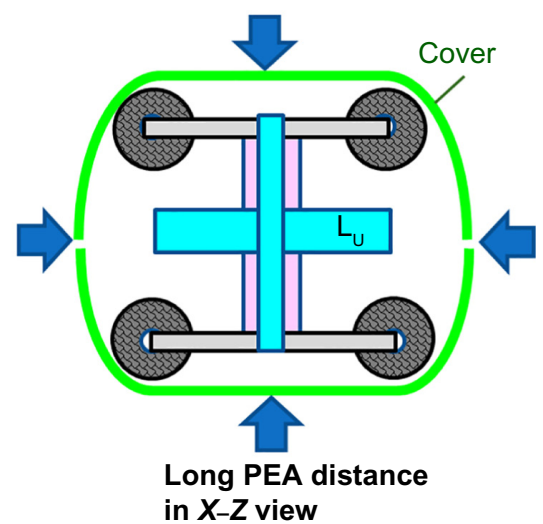

Figure $7 J_{3}$ antagonistic mechanism and $L_{4}$ with cover.

Notes: (A) Short PEA distance in $X$ direction in $X-Z$ view; (B) long PEA distance in $X$ direction in $X-Z$ view. See Table I for definitions of symbols. Abbreviation: PEA, pneumatic elastic actuator.

A

Only straight lines, A

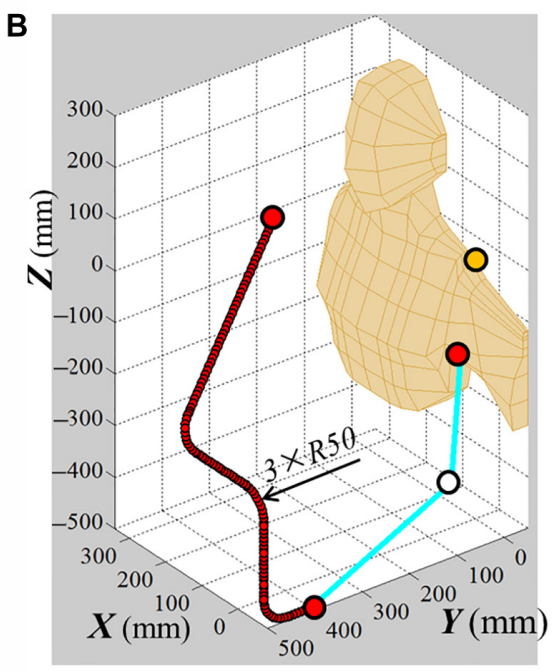

A with round $\mathrm{R} 50$ at corners, $\mathrm{B}$
End point

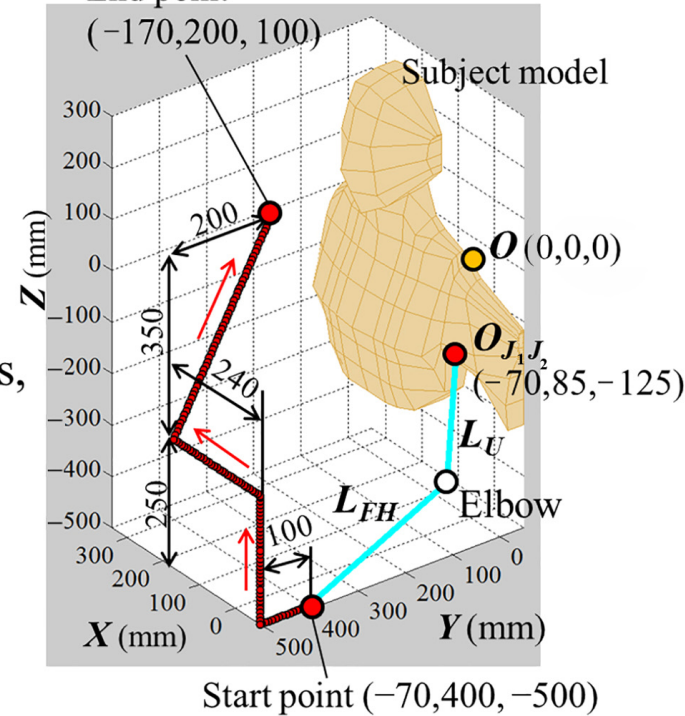

Start point $(-70,400,-500)$



Smoothed B by moving average, $\mathrm{C}$

Figure 8 Comparison of three pathways.

Notes: (A) Pathway A; (B) pathway B; (C) pathway C. See Table I for definitions of symbols. 
was based on typical ADL hand-to-mouth movements in the horizontal and vertical direction, as shown in Figure 8. The first plot (Figure 8A) shows the pathway formed with straight lines (pathway A). A constraint condition was set such that the axis $Y_{H}$ of the coordinate system of the hand in Figure $5 \mathrm{~A}$ and $\mathrm{B}$ was always in the sagittal plane (the $Y-Z$ plane with an arbitrary $X$ position). This permits the hand to easily grasp an object, such as a bottle on a table. The velocity of the hand and the time interval of the points on the pathway were set to $0.45 \mathrm{~m} / \mathrm{sec}$ and $0.0167 \mathrm{sec}$, respectively, based on observations of human ADL motion and the sampling frequency of $1 / 60 \mathrm{~Hz}$ of the motion capture system used in our previous study. ${ }^{26}$

Because the torque was assumed to increase rapidly because of the dramatic change in the direction of the straight line at the corners in pathway A, another pathway (pathway B) was established in which the sharp corners were replaced with curves having radii of $50 \mathrm{~mm}$, as shown in Figure 8B. A third pathway (pathway C) was established with the curves smoothed using a moving average of 10 points and three time repetitions as shown in Figure 8C. The required joint angle $\phi$ and torque $T$ were simulated using equations (2) and (3) for the arm tracking each of these pathways. These results were used to select the configuration of the PEAs in the AM, ie, to determine $n_{s}$ and $n_{p}$ using equation (1) and equations (4) to (6).

\section{Results \\ Experiments}

\section{Spring constant}

The relationship between the shrinkage length $x_{s l}$ with respect to the unloaded natural length of the PEA and the traction force $F_{t}$ per PEA piece during actuation at $0.2 \mathrm{MPa}$ is shown in Figure 9. The blue and red curves correspond to the cases of actuation (application of air pressure) after adding the weight and adding the weight after actuation,

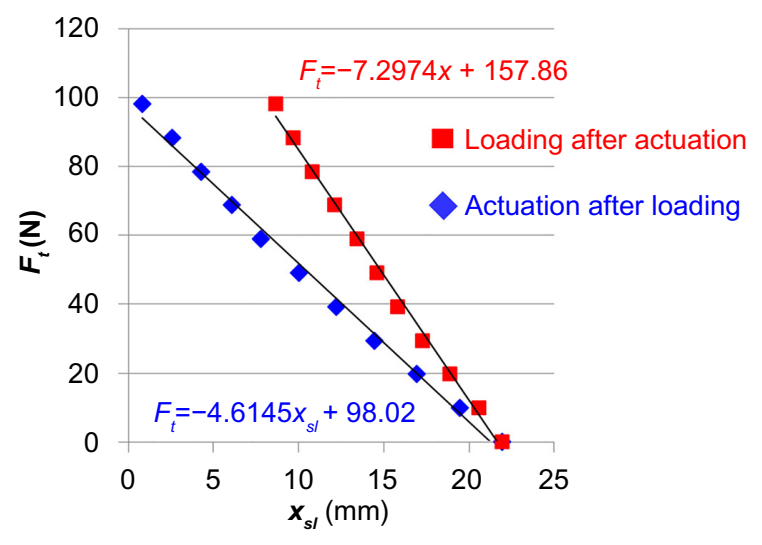

Figure 9 Relationship between $x_{s l}$ and $F_{t}$ at $0.2 \mathrm{MPa}$. respectively. The measurements were repeated ten times. There were differences between the two cases due to the influence of hysteresis. The stricter conditions represented by the blue curve were considered in the calculation of the spring constant. Equations (1) and (6) were modified to reflect the measurement results indicated by the blue curve. Based on equation (7), the maximum pull force of each PEA is $98.02 \mathrm{~N}$ when fully extended $\left(x_{s l}=0\right)$ although the one in its product catalog 23 is $100 \mathrm{~N}$.

$$
\begin{gathered}
F_{t}=-4.61 x_{s l}+98.02 \\
T_{A M}=F_{t} r=n_{p}\left(-4.61 x_{s l} / n_{s}+98.02\right) r
\end{gathered}
$$

\section{Impact absorption in direction around long axis of PEA}

The results of the impact tests in the direction around the long axis of the PEA are shown in Figure 10A. The test was conducted ten times. The graph shows the peak values of the force for air pressures of $0.05,0.1$, and $0.2 \mathrm{MPa}$, as shown in Figure 3A, and without the PEA (direct collision), as shown in Figure 3B. For the cases of an air pressure of $0.2 \mathrm{MPa}$ and direct impact, the results for weights from 0.98 to $9.8 \mathrm{~N}$ $(1 \mathrm{~kg})$ are shown in Figure 10B. The results with and without the PEA are significantly different $(P<0.01)$ for all six weight levels. Therefore, the requirement for PEAs placed around the link (the base frame) was confirmed as a basic principle, as described in the section outlining the method for determining PEA configuration in AM and layout of PEA and motor in each joint.

\section{Impact absorption in direction of long axis of PEA in AM versus motor}

The results of the impact tests in the direction of the long axis of the PEA in the AM are compared with those for the motor in Figure 11. The tests were repeated ten times. Figure 11 shows the peak values of the force on the arm with the PEA in the AM for air pressures of $0.05,0.1$, and $0.2 \mathrm{MPa}$, as shown in Figure 4D, and for the motor, as shown in Figure 4E. The air pressure of the PEA in the AM was initially set to $0.2 \mathrm{MPa}$. To achieve a torque of the AM at an air pressure of $0.2 \mathrm{MPa}$ close to that of the motor $(4.70 \mathrm{~N} \cdot \mathrm{m})$, two PEAs were used in parallel on each side $\left(n_{s}=1\right.$ and $\left.n_{p}=2\right)$. The AM torque was calculated to be $4.74 \mathrm{~N} \cdot \mathrm{m}$ (for $r=30 \mathrm{~mm}$ ). The air pressure was subsequently decreased to $0.1 \mathrm{MPa}$ and then $0.05 \mathrm{MPa}$. The peak values of the force on the arm with the PEA in the AM were decreased in comparison with the motor. The results between each PEA and the motor are significantly different $(P<0.01)$. 
A

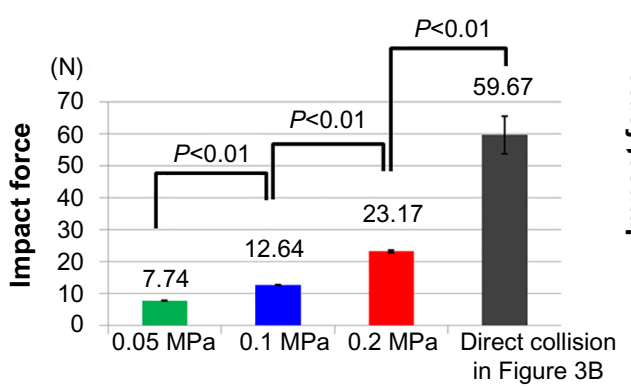

B

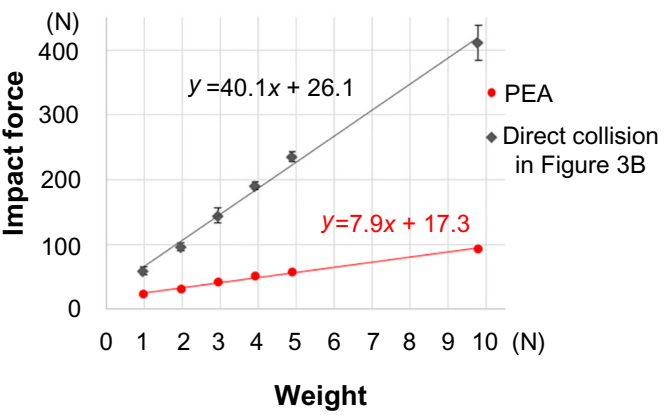

Figure 10 Comparison of peak impact force: with and without PEA in direction around long axis.

Notes: (A) Comparison of impact force between PEA $(0.05,0.1,0.2 \mathrm{MPa})$ and direct collision in Figure $3 \mathrm{~B}$ by weight $0.98 \mathrm{~N}$; (B) comparison of impact force between PEA $(0.2 \mathrm{MPa})$ and direct collision in Figure $3 \mathrm{~B}$ by weight $0.98,1.96,2.94,3.92,4.9,9.8 \mathrm{~N}$.

Abbreviation: PEA, pneumatic elastic actuator.

\section{Impact absorption in direction of long axis of PEA} in $A M$ versus motor with and without PEA on arm

The results of the impact testing illustrated in Figure 4F and $4 \mathrm{G}$ are shown in Figure 11B. The AM joint of PEAs with the PEA on the arm (shown in Figure 4F) reduced the force to $87.4 \%$ of that of the same joint without the PEA (shown in Figure 4D). The motor joint with the PEA (Figure 4G) also reduced the force to $67.8 \%$ of that of the joint without the PEA (Figure 4E).

\section{Modeling}

\section{Required torque and angle of joints}

Figure 12A-D shows the required torques $T\left(T_{1}, T_{2}, T_{3}, T_{4}\right)$ and angles $\phi\left(\phi_{1}, \phi_{2}, \phi_{3}, \phi_{4}\right)$ of the joints associated with following the three pathways shown in Figure 8: with straight lines only (pathway A); with curves rounded and a radii of $50 \mathrm{~mm}$ (pathway B); and with smoothing performed using a moving average (pathway $\mathrm{C}$ ). The maximum absolute val- ues of the torques for pathway $\mathrm{C}$ were smaller for all of the joints than those for pathways A and B, as shown in Figure $12 \mathrm{E}$. Therefore, pathway $\mathrm{C}$ was used to simulate the configuration of the AM using equations (2), (3), and (8).

\section{Configuration of $\mathrm{AM}$ and motor layout in each joint}

Based on the results for pathway $\mathrm{C}$ described in the section on required torque and angle of joints, the configuration of the AM in each joint was designed using equations (2), (3), and (8), as shown in Table 2. The values of $T, T_{A M}$, and $\phi$ are shown in Figure 13A-D. In Table 2, the number of required actuators is the total for both the antagonist and agonist sides in Figure 6. Both positive and negative torques $T_{1}$ and $T_{4}$ in the coordinate system shown in Figure 5A were calculated. Absolute values of $T_{1}$ and $T_{4}$ are shown for comparison with $T_{A M 1}$ and $T_{A M 4}$ in Figure 13A and D. There are places at which $T_{A M 1}$ and $T_{A M 4}$ change suddenly as a result of a change in the side (antagonist or agonist in Figure 6) that should be
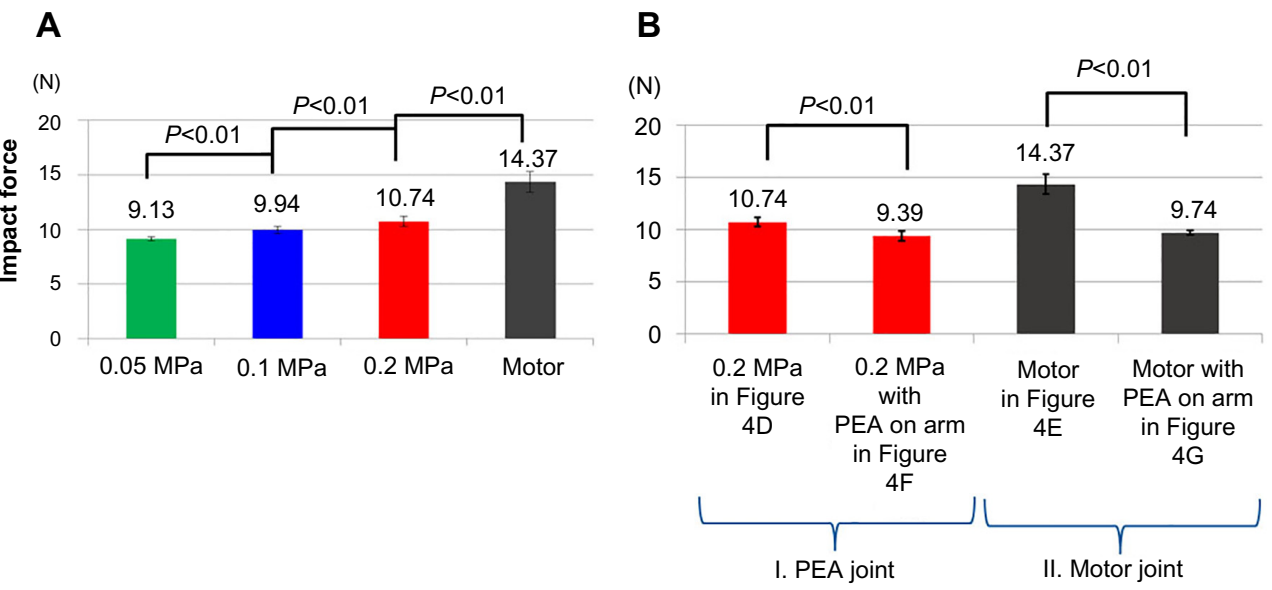

Figure I I Comparison of peak impact force at joint consisting of PEAs at each pressure and of motor with and without PEA on arm.

Notes: (A) Comparison of impact force between PEA (0.05, 0.1, $0.2 \mathrm{MPa})$ and motor in joint; (B) comparison of effect of PEA on arm between PEA (0.2 MPa) joint and motor joint.

Abbreviation: PEA, pneumatic elastic actuator. 
A

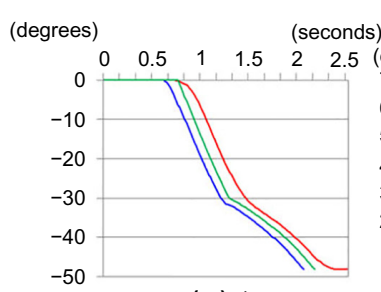

$\left(a_{1}\right) \phi_{1}$



$\left(a_{2}\right) T_{1}$
B

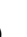

)



(Nm)

$\left(b_{1}\right) \phi_{2}$

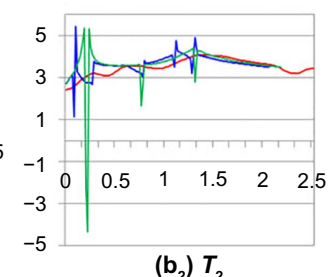

(b) $T_{2}$
C
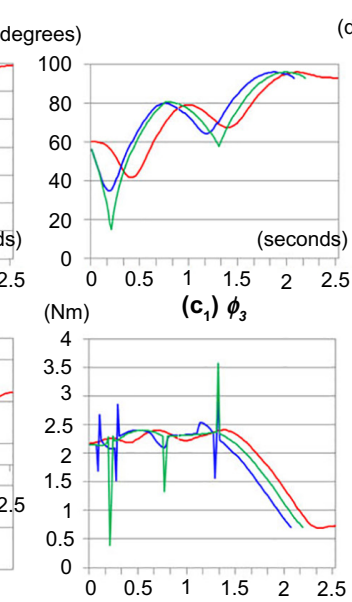

(c) $T_{3}$
D

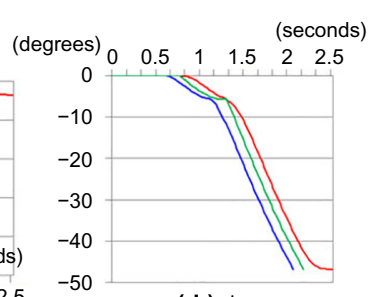

$\left(d_{1}\right) \phi_{4}$



(d) $T_{4}$
E

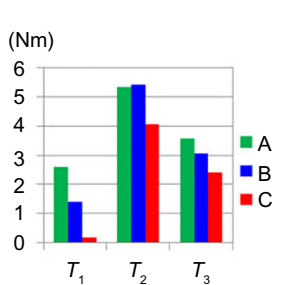

(Nm) 0.03



Maximum absolute values of $T_{1-4}$

Pathway A

Pathway B

Pathway C

Figure 12 Comparison of required joint angle and torque in pathways (A-C).

Notes: (A) $\varphi_{1}$ and $T_{1}$ in $J_{1} ;\left(\right.$ B) $\varphi_{2}$ and $T_{2}$ in $J_{2} ;$ (C) $\varphi_{3}$ and $T_{3}$ in $J_{3} ;$ (D) $\varphi_{4}$ and $T_{4}$ in $J_{4} ;\left(\right.$ E) maximum absolute values of $T_{1}, T_{2}, T_{3}$, and $T_{4}$. See Table I for definitions of symbols.

activated. The AMs of $J_{1,2,3}$ (but not $J_{4}$ ) were set to rotate in one direction only with respect to the initial posture in the coordinate system shown in Figure 5A because the rotational direction of $\phi_{1,2,3}$ in Figure 5A is only in one direction $\left(\phi_{1},-\right.$; $\phi_{2},+; \phi_{3},+; \phi_{4}, \pm$ ) as indicated by the "Required $\phi$ " in Table 2. On the other hand, because of the required rotational angle $\phi_{4}$ in both the positive and negative directions for $\mathrm{J}_{4}$, the $\mathrm{AM}$ of $\mathrm{J}_{4}$ was set to rotate in both directions. Therefore, the constraint for $\mathrm{J}_{4}$ was strict in comparison with that for $\mathrm{J}_{1,2,3}$, and a special method was employed only for $\mathrm{J}_{4}$. The absolute maximum rotational angle of the $\mathrm{AM}$ of $\mathrm{J}_{4}$, based on the configuration in Table 2, was initially $40.61^{\circ}$, which was less than the absolute maximum required $\phi_{4}$ of $46.75^{\circ}$. Thus, a gear train with a speed reduction ratio of 0.5 was employed to address the situation of $\mathrm{J}_{4}$. The absolute maximum angle of the $\mathrm{AM}$ of $\mathrm{J}_{4}$ was thereby increased to $81.22^{\circ}$, which exceeded the $\phi_{4}$ value of $46.75^{\circ}$. In turn, although the torque was reduced, the averages of the absolute values of $T_{A M 4}$ with the gear and $T_{4}$ were $0.8365 \mathrm{Nm}$ and 0.0003 $\mathrm{Nm}$, respectively, and the $T_{A M 4}$ of the configuration met the requirement for $T_{4}$. The total weight of the gear train and the bevel-gear drive box was $35 \mathrm{~g}$.

Based on these results, because the total weight of the AM with the PEAs of $J_{2}$ exceeded $0.134 \mathrm{~kg}$, ie, the threshold selected as described in the section on the method for determining PEA configuration in AM and layout of PEA and motor in each joint, the motor drive was selected as a substitute for the AM only for $\mathrm{J}_{2}$.

\section{Design and simulation of 4-DOF arm}

Based on the results described in the previous section, the 4-DOF arm shown in Figure 14A-C was designed. Because of the significant differences in the results shown in the section on impact absorption in direction around the long axis of PEA, and Figures 3 and 10 were confirmed, PEAs were placed around the link $\mathrm{L}_{\mathrm{U}}$ and $\mathrm{L}_{\mathrm{FH}}$ in the 4-DOF arm design. The PEAs of the AMs of $\mathrm{J}_{3}$ and $\mathrm{J}_{4}$ were placed on $\mathrm{L}_{\mathrm{U}}$ and $\mathrm{L}_{\mathrm{FH}}$, respectively. Because neither $\mathrm{J}_{3}$ nor $\mathrm{J}_{4}$ had $n_{s}>2$, this placement was feasible, as described in the section on the method for determining PEA configura-

Table 2 Required number of actuators

\begin{tabular}{|c|c|c|c|c|c|c|c|c|c|c|c|c|}
\hline \multirow[t]{2}{*}{ Joint } & \multirow{2}{*}{$\begin{array}{l}\text { One PEA } \\
\text { maximum } \\
\text { pull force }(\mathbf{N})\end{array}$} & \multirow{2}{*}{$\begin{array}{l}\text { Number of } \\
\text { required } \\
\text { actuators }\end{array}$} & \multicolumn{2}{|c|}{ Combination } & \multicolumn{2}{|c|}{$\begin{array}{l}\text { Required } \phi \\
\text { (degrees) }\end{array}$} & \multirow{2}{*}{$\begin{array}{l}\text { Absolute } \\
\text { maximum } \phi \text { of } \\
\text { AM (degree) }\end{array}$} & \multirow{2}{*}{$\begin{array}{l}\text { Total } \\
\text { PEAs } \\
(\mathrm{kg})\end{array}$} & \multirow{2}{*}{$\begin{array}{l}\text { Pulley } \\
\text { weight } \\
\text { (kg) }\end{array}$} & \multirow[t]{2}{*}{$\begin{array}{l}\text { Accessory } \\
(\mathrm{kg})\end{array}$} & \multirow[t]{2}{*}{$\begin{array}{l}\text { Gears } \\
(\mathrm{kg})\end{array}$} & \multirow{2}{*}{$\begin{array}{l}\text { Total } \\
\text { weight } \\
\text { (kg) }\end{array}$} \\
\hline & & & $n_{p}$ & $\overline{n_{s}}$ & $\min \phi$ & $\max \phi$ & & & & & & \\
\hline$J_{1}$ & 98.02 & 2 & I & I & -48.15 & 0.00 & 81.22 & 0.006 & 0.01 & 0.01 & & 0.026 \\
\hline $\mathrm{J}_{2}$ & $(0.2 \mathrm{MPa})$ & 20 & 5 & 2 & 4.79 & 69.13 & 162.43 & 0.060 & 0.01 & 0.1 & & 0.170 \\
\hline $\mathrm{J}_{3}$ & & 12 & 3 & 2 & 41.77 & 96.01 & 162.43 & 0.036 & 0.01 & 0.06 & & 0.106 \\
\hline $\mathrm{J}_{4}$ & & 4 & 2 & I & -46.75 & 0.001 & 81.22 & 0.012 & 0.01 & 0.02 & 0.035 & 0.077 \\
\hline
\end{tabular}

Abbreviations: AM, antagonistic mechanism; PEAs, pneumatic elastic actuators. 

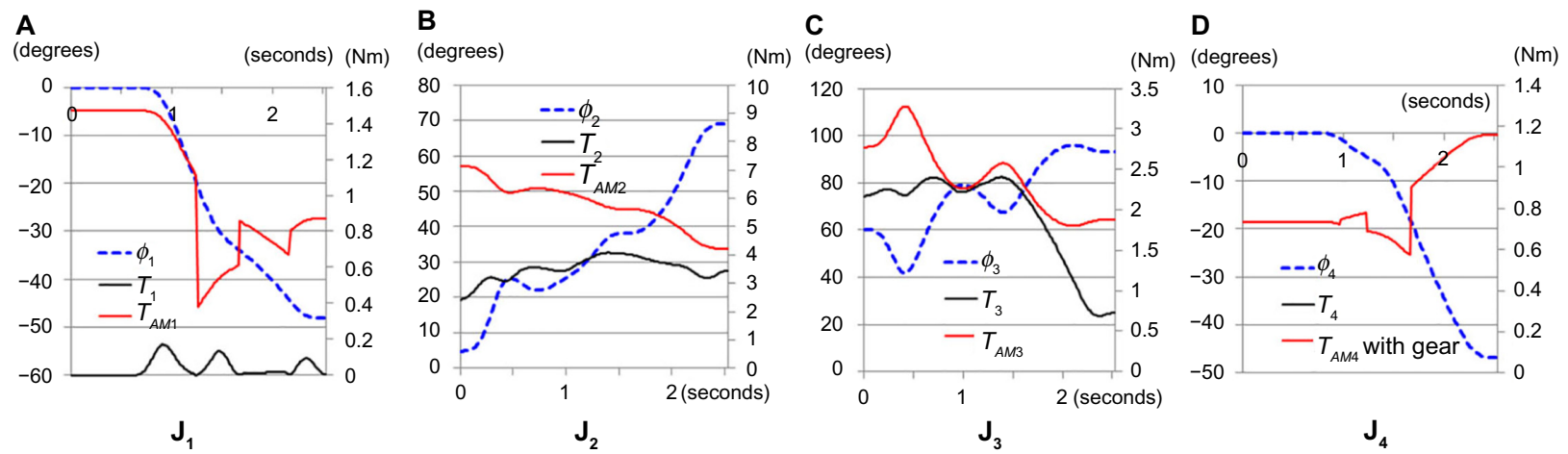

Figure 13 Torque and joint angles.

Notes: $(\mathbf{A}) \varphi_{1}, T_{1}$, and $T_{A M 1}$ in $J_{1} ;(\mathbf{B}) \varphi_{2}, T_{2}$, and $T_{A M 2}$ in $J_{2} ;(\mathbf{C}) \varphi_{3}, T_{3}$, and $T_{A M 3}$ in $J_{3} ;(\mathbf{D}) \varphi_{4}, T_{4}$, and $T_{A M 4}$ with gear in $J_{4}$. See Table I for definitions of symbols.

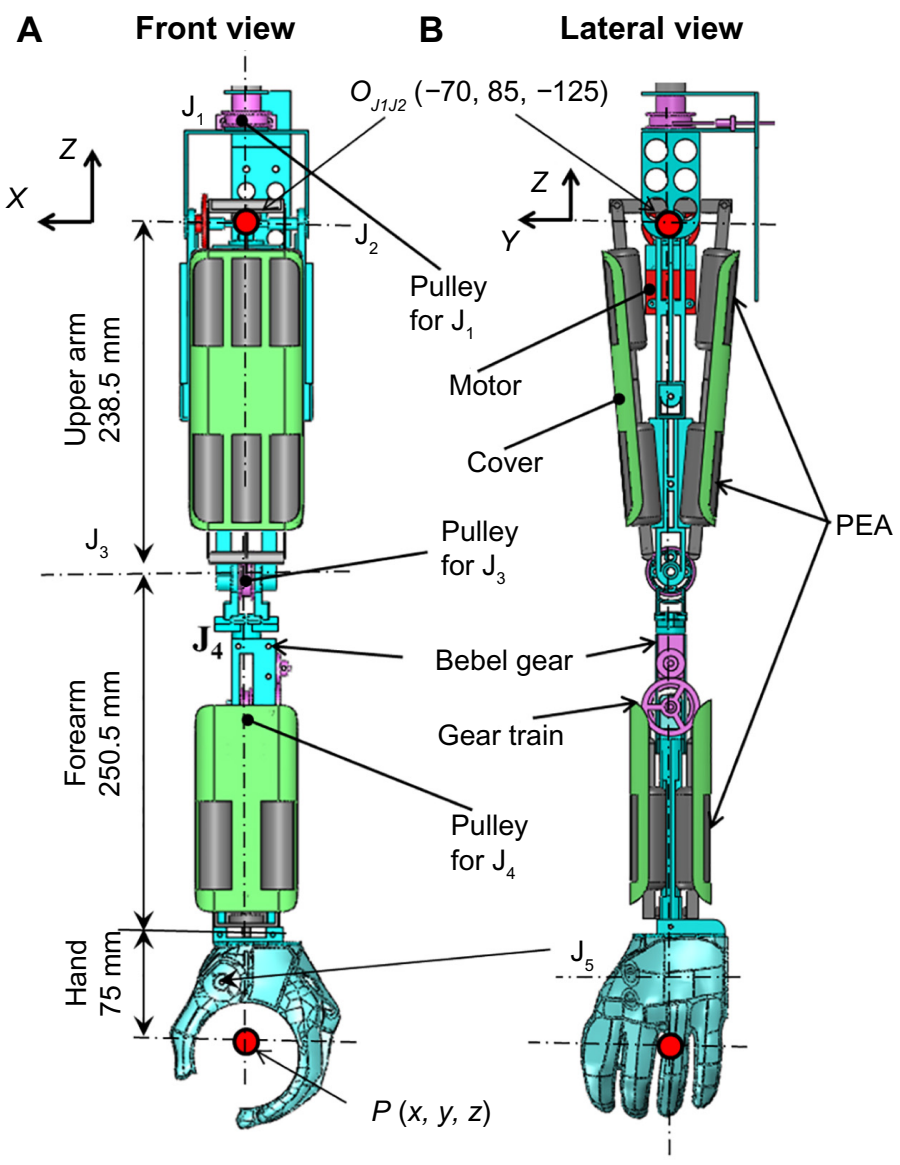

C Arm with cover in horizontal posture $\left(\phi_{2}=90^{\circ}\right)$ and impact force

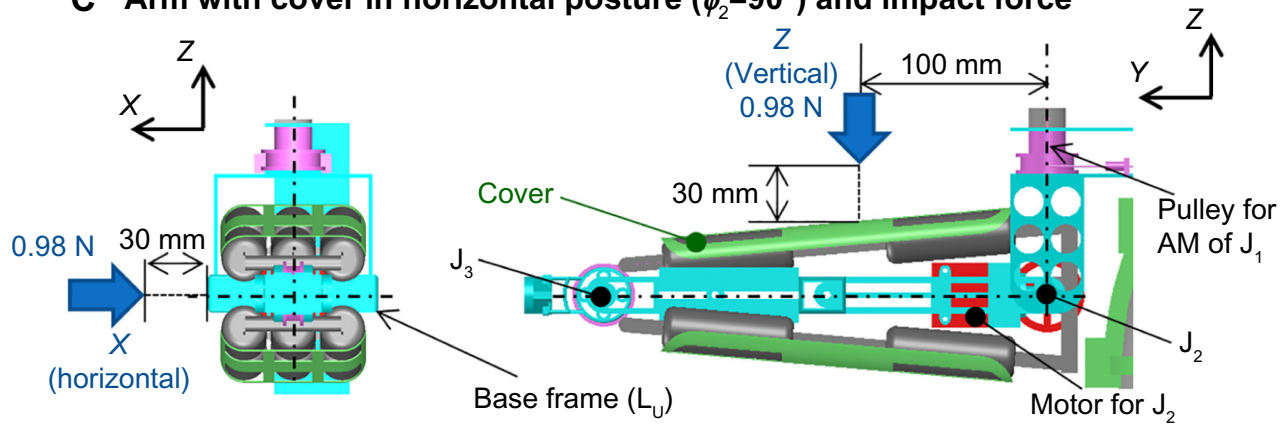

Figure 14 Design of 4-DOF arm with I-DOF hand based on the results of determining PEA configuration in AM, and layout of PEA and motor in each joint.

Notes: (A) 4-DOF arm in front view; (B) 4-DOF arm in lateral view; (C) arm in horizontal posture $\left(\varphi_{2}=90^{\circ}\right)$ and impact force. See Table I for definitions of symbols. Abbreviations: AM, antagonistic mechanism; PEA, pneumatic elastic actuator; 4-DOF, four degrees-of-freedom; I-DOF, single degree-of-freedom. 
tion in AM and layout of PEA and motor in each joint. The PEAs of the AM of $\mathrm{J}_{1}$ were placed on the backpack. The 1-DOF motor-driven hand with grasping capability $\left(\mathrm{J}_{5}\right.$ in Figure 14B), which was prototyped previously, ${ }^{26}$ was incorporated into the design to make the shoulder prosthesis complete. The motors for the hand were also assumed to be set in the backpack.

As shown in Figure 14C, the output impact forces on the shoulder with respect to the input impact forces to the link $\mathrm{L}_{\mathrm{U}}$ in the horizontal $(X)$ and vertical $(Z)$ directions in the case of the entire arm extending in the $Y$ direction $\left(\phi_{2}=90^{\circ}\right)$ were estimated as shown in Table 3 , based on the result described in the section on impact absorption in the direction of the long axis of PEA in AM versus motor with and without PEA on the arm and Figure 11B. Estimates based on the layout described in Table 2 and for layouts composed of all PEA joints and all motor joints are shown for comparison. Because the upper $\operatorname{arm} \mathrm{L}_{\mathrm{U}}$ was not designed as shown in Figure $7 \mathrm{~B}$ but rather as shown in Figure 7A, the impact on the shoulder in the $X$ direction in Figure 14C was estimated without the effect of impact absorption by the PEA around its long axis direction. This case corresponds to the results labeled " $0.2 \mathrm{MPa}$ in Figure $4 \mathrm{D}$ " in Figure 11B.

The weight of the arm in the simulation is shown in Table 4. For all components, except the hand and back pack, the weight calculated by CAD was used. For the hand and backpack, the weight of actual parts, manufactured for another project, was used. The arm weight could be estimated to be lower than $1 \mathrm{~kg}$.

\section{Discussion}

In this paper, the torque, impact absorption, and ROM of a shoulder prosthesis were investigated by both experiments and simulation. Two different types of actuators embedded in a 1-DOF arm were compared for the purpose of developing a shoulder prosthesis in which an adequate working range, output force, and impact absorption capacity were provided in a balanced manner based on the layout of the actuators. The characteristics of the PEAs used in the prosthesis were measured.

Table 3 Impact force on shoulder in simulation

\begin{tabular}{llll}
\hline $\begin{array}{l}\text { Force } \\
\text { direction }\end{array}$ & $\mathbf{N}$ & & \\
\cline { 2 - 4 } & Joint & & \\
\cline { 2 - 4 } & Result in Table 2 & All PEA & All motor \\
\hline X (horizontal) & 10.74 & 9.39 & 14.37 \\
$Z$ (vertical) & 9.47 & 9.39 & 14.37 \\
\hline
\end{tabular}

Abbreviation: PEA, pneumatic elastic actuator.

\section{Experiments}

The graph of the traction force $F_{t}$ and the shrinkage length $x_{s l}$ in Figure 9 applies to the case of an air pressure fixed at $0.2 \mathrm{MPa}$. The relationship between $F_{t}$ and $x_{s l}$ is approximately linear. Although $T$ and $T_{A M}$ were calculated based on this result and equation (1), the derivation of the relationship over the entire pressure range considered, eg, for increments of $0.01 \mathrm{MPa}$, would be desirable to improve the accuracy of the results. The next practical step would be to establish the parameters for a given air pressure.

With respect to impact absorption, the impact force in the direction around the long axis of the PEA (on the external surface) was found to be greatly decreased in comparison with a direct impact on the measurement surface of the force gauge, as shown in Figures 3 and 10. This trend continued with increasing weight. As shown in Figure 10B, the impact force with the PEA was approximately $20 \%$ of that of the direct collision. Therefore, use of PEAs can contribute to safe use of the prosthesis, and the design should include PEAs placed around the arm. Moreover, the impact force could be decreased by decreasing the air pressure. The relationship between air pressure, shrinkage length $x_{s l}$, and degree of impact absorption, ie, the relationship between the joint angle, arm position, posture, and vulnerability to impact, should be studied further. The impact absorption capacity in the axial direction of the PEA in the AM with the 1-DOF arm was shown to be greater than that of the motor joint (see Figure 4D and E) and to vary with air pressure, as shown in Figure 11A. Although a motor can generate pseudocompliance through motor control, the associated time delay is an issue. The intrinsic compliance of the actuator eliminates the time delay in absorbing an impact. To confirm this, continuous impact data along the temporal axis should be measured, and the influence of the time delay should be investigated. Moreover, the measured value for the $0.2 \mathrm{MPa}$ PEA $(10.74 \mathrm{~N})$ and the value for direct collision $(14.37 \mathrm{~N})$ in Figure 11A was significantly decreased in comparison with the result $(59.67 \mathrm{~N})$ in Figure 10A under the same condition of input force in collision with the rigid part (the arm or measurement site of the force gauge) in Figures 3B, 4D, and E. This is considered to be due to impact absorption by the joint and main body of the arm. Thus, the impact force is considered to increase as the collision position approaches the joint, ie, verging on the condition shown in Figure 3B. The shape of the main body of the arm must be validated. Further, the difference between the forces with and without the PEA on the arm (see Figures 4D-G and 11B) was not large in comparison with the difference between the force with the 0.2 MPa PEA and the direct collision (see Figure 10). However, 
Table 4 Arm weight in simulation

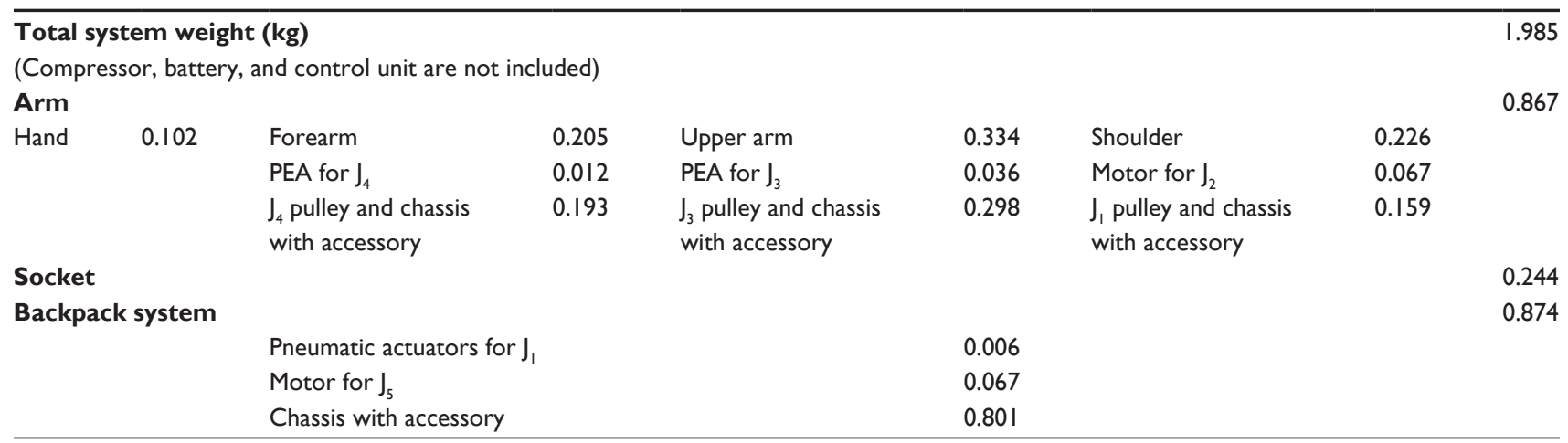

Notes: See Table I for definitions of symbols.

Abbreviation: PEA, pneumatic elastic actuator.

the difference between the motor joint with and without the PEA was greater than that between the PEA joint with and without the PEA on the arm, as shown in Figure 11B. Therefore, even if a certain joint is driven by the motor, the impact is considered to be decreased by covering the arm body with the PEA of the other joint. The results shown in Figure $14 \mathrm{C}$ and Table 3 indicate that the design could not absorb the impact force in the $X$ direction using the external surface of the PEA. However, the force decreased in comparison with all of the motor joints as a whole because $\mathrm{J}_{1}$ was the PEA joint, as shown in Table 3. The effect of impact absorption could be confirmed by employing either a PEA joint or by placing PEAs around the arm.

\section{Modeling}

We next consider the results related to the pathways considered and the joint behavior while following the pathways. Using equations (2) and (3), the required angle $\phi$ and torque $T$ were calculated for the three types of pathways, as shown in Figure 12, and $T$ was shown to be decreased by smoothing the pathway, as shown in Figure 12E. This indicates that planning the path of a motion affects the ideal layout of the actuators in the arms for a given motion. This problem also affects the output force of the actuator and the supply source (battery or air compressor) used, and thus should be taken into consideration.

In this paper, the threshold for selecting either the AM or motor was set to the weight of two motors $(0.134 \mathrm{~kg})$ based on one motor weighing $0.067 \mathrm{~kg}$. In this way, the motor and the AM of the PEA were selected for $\mathrm{J}_{2}$ and the other joints, respectively. However, the selection is highly dependent on the threshold. For example, if the weight of one motor $(0.067 \mathrm{~kg})$ were used as the threshold, the AM of the PEA would only be selected for $\mathrm{J}_{1}$; if the weight of three motors $(0.201 \mathrm{~kg})$ were used as the threshold, the AM of the PEA would be selected for all of the joints. Moreover, although the pulley radius $r$ for all of the joints was set to $15 \mathrm{~mm}$, the $T_{A M}$ changes with $r$, as shown in equation (8); thus, the layout selection may change as the threshold changes. Further, the relationship between number of actuator connections $\left(n_{s}\right.$ and $n_{p}$ ) and $r$ is seemingly simple (for example, the value of $T_{A M}$ when $n_{p}$ doubles seems to be equal to the value of $T_{A M}$ when $r$ doubles, for a fixed value of $n_{s}$ ). However, because $r$ is present twice in equations (4) and (8), ie. in $r^{2}$ as well, the relationship becomes complex and should be investigated carefully. In this study, although the ratio of $T_{A M}$ to $T$ was not considered, a factor of safety should be established. These factors should be considered in further optimization of the design.

In joint $\mathrm{J}_{4}$, a gear train with a speed reduction ratio of 0.5 was used. A PEA can exert large force when $x_{s l}$ is small, as shown in Figure 9. Therefore, the PEA is considered to be of greater advantage for small ranges of motion. For example, in the case of $\phi_{4}=20^{\circ}$ without the gear and $r=15 \mathrm{~mm}, T_{A M 4}$ was calculated to $0.746 \mathrm{Nm}$. When the gear train was mounted and the angle of the output shaft of the train was set to $\phi_{4}=20^{\circ}$, the required input pulley angle was only $10^{\circ}$, and the calculated $T_{A M 4}$ was $0.554 \mathrm{Nm}$. Although the output angle and speed are inversely proportional to the output torque in a normal gear train, in this case the output torque was greater than $50 \%(0.554 / 0.746=74.26 \%)$ when the output angle doubled. In the case of $\phi_{4}=30^{\circ}$, the output $T_{A M 4}$ without and with the gear (for an input pulley angle of $15^{\circ}$ ) was $0.384 \mathrm{Nm}$ and $0.464 \mathrm{Nm}$, respectively. That means that the output angle remained unchanged, and the output torque improved to $0.464 / 0.384=120.83 \%$. Installation of a gear train has a disadvantage in that it increases the weight and may delay the response. However, this can be a useful trade-off depending on the rotational range.

\section{Conclusion}

In this study, a lightweight hybrid-type shoulder prosthesis in which PEAs are combined with a motor was designed 
to achieve an adequate working range, output force, and impact absorption capacity in a balanced manner. To design AMs of PEAs that have the potential to enhance the safety of prosthetic joints, the $F_{t}-x_{s l}$ relationship of the PEA was determined experimentally at the beginning of the study. A measuring system with a 1-DOF arm for use in investigating the feature of the two types of actuators was built, and the impact absorption capacity in both the long axial direction and around the long axis direction of the PEA was measured in collision experiments and compared with that of a motor. The results show that arranging PEAs throughout the arm makes it possible to achieve sufficient impact absorption capacity both inside the joints and on the external surface of the arm. This could reduce the impact force by approximately $35 \%$, compared with all-motor configuration, as shown in Table 3.

A link model was developed and dynamic equations were derived to simulate the required angle $\phi$ and torque $T$ as the arm tracked pathways corresponding to a typical ADL hand-to-mouth movement. The equation for the $F_{t}-x_{s l}$ relationship was used to develop a procedure for determining the required number of PEAs in the AM $\left(n_{s}\right.$ and $\left.n_{p}\right)$. Finally, the actuator (PEA or motor) to be used in each shoulder and elbow joint was determined using a threshold based on the weight of the motor; the PEAs and motor were set for $\mathrm{J}_{1}-\mathrm{J}_{3}-\mathrm{J}_{4}$ $\left[n_{s}(1,-, 2,1)\right.$ and $\left.n_{p}(1,-, 3,2)\right]$ and $\mathrm{J}_{2}$, respectively, as shown in Table 2. A CAD model was designed on the basis of the results of the layout analysis and collision experiments, and 10.74 and $9.47 \mathrm{~N}$ of the impact forces on the shoulder were estimated by applying a collision force to the upper arm in the horizontal and vertical directions against $14.37 \mathrm{~N}$ of all motor layout, respectively, as shown in Table 3. The arm weight could be estimated within $1 \mathrm{~kg}$, as shown in Table 4. The problem of the trade-off between the output torque and the output angle was addressed by installing a gear train in the AM of one of the joints.

\section{Future work}

In this paper, although the simulation used typical pathways, various ADL motions of actual subjects should be measured, and those trajectories should be reproduced with a real prosthetic arm. Then, by examining the relationship between the various ADL movements and the optimal layouts of the actuators in the joints, useful information can be provided to other researchers and prosthetists concerning not only the required torques and angles to be simulated but also suitable actuator types for various ADL movements.
The dimensions and specifications of a prosthesis vary depending on the body habitus and the ADL movements needed by the user. Optimizing the pulley radius, gear ratio, and number and configuration of PEAs used is a future challenge in the development of the proposed prosthesis model. There is also an urgent need to establish control techniques to utilize these elements.

\section{Acknowledgment}

This work was primarily supported by the Japan Society for the Promotion of Science Grant-in-Aid for Scientific Research (B), Grant Number 26282160.

\section{Disclosure}

The authors declare no conflicts of interest in this work.

\section{References}

1. Ohnishi K. [Improving the usability of myoelectric controls and upper limb prostheses]. Bulletin of the Japanese Society of Prosthetics and Orthotics. 2011;27:84-88. Japanese.

2. Polisiero M, Bifulco P, Liccardo A, et al. Design and assessment of a low-cost, electromyographically controlled, prosthetic hand. Medical Devices: Evidence and Research. 2013;6:97-104.

3. Peerdeman B, Smit G, Stramigioli S, Plettenburg DH, Misra S. Evaluation of pneumatic cylinder actuators for hand prostheses. In: Proceedings of the Fourth IEEE RAS and EMBS International Conference on Biomedical Robotics and Biomechatronics. 2012;1104-1109.

4. Gonzalez J, Soma H, Sekine M, Yu W. Psycho-physiological assessment of a prosthetic hand sensory feedback system based on an auditory display: a preliminary study. J Neuroeng Rehabil. 2012;9:1-14.

5. Zhang T, Fan S, Zhao J, et al. Design and control of a multisensory five-finger prosthetic hand. In: Proceedings of the 11th World Congress on Intelligent Control and Automation. 2014:3327-3332.

6. Low CY, Kasim MAA, Koch T, et al. Hybrid-actuated finger prosthesis with tactile sensing. International Journal of Advanced Robotic Systems. 2013;10:1-10.

7. Yong X, Jing X, Jiang Y, et al. Tendon drive finger mechanisms for an EMG prosthetic hand with two motors. In: Proceedings of Seventh International Conference on Biomedical Engineering and Informatics. 2014:568-572.

8. Sittiwanchai T, Nakayama I, Inoue S, et al. Transhumeral prosthesis prototype with 3D printing and sEMG-based elbow joint control method. In: Proceedings of the 2014 International Conference on Advanced Mechatronic Systems. 2014:227-231.

9. Fite KB, Withrow TJ, Shen X, et al. A gas-actuated anthropomorphic prosthesis for transhumeral amputees. IEEE Trans Robot. 2008;24: 159-169.

10. Kundu SK, Kiguchi K. Development of a 5 DOF prosthetic arm for above elbow amputees. In: Proceedings of IEEE International Conference on Mechatronics and Automation. 2008:207-212.

11. Yoshikawa M, Taguchi Y, Sakamoto S, et al. Trans-radial prosthesis with three opposed fingers. In: Proceedings of the 2013 IEEE/RSJ International Conference on Intelligent Robots and Systems. 2013: 1493-1498.

12. Okamoto S, Tamura T, Koike M, et al. [Survey of attitude for amputees of hemi lateral forearm to artificial electric arm]. Research Bulletin National Rehabilitation Center for Persons with Disabilities. 2001;22: 55-61. Japanese.

13. Resnik L, Klinger SL, Etter K. The DEKA arm: its features, functionality, and evolution during the Veterans Affairs study to optimize the DEKA arm. Prosthet Orthot Int. 2014;38:492-504. 
14. Miller LA, Lipschutz RD, Stubblefield KA, et al. Control of a six degree of freedom prosthetic arm after targeted muscle reinnervation surgery. Arch Phys Med Rehabil. 2008;89:2057-2065.

15. Fukaya N, Sawada K, Oku H, et al. [Development of an artificial arm by using a spherical ultrasonic motor]. Journal of the Japan Society for Precision Engineering. 2001;67:654-659. Japanese.

16. Yokoyama O, Takakura T, Itoh R, et al. [A case report of a high level of upper limb amputee with myoelectric hand]. Bulletin of the Japanese Society of Prosthetics and Orthotics. 2009;25:156-159. Japanese.

17. Xu B, Peng S, Song A, et al. Robot-aided upper-limb rehabilitation based on motor imagery EEG. International Journal of Advanced Robotic Systems. 2011;8:88-97.

18. Laurin-Kovitz KF, Colgate JE, Carnes SDR. Design of components for programmable passive impedance. In: Proceedings of IEEE International Conference on Robotics and Automation. 1991: 1476-1481.

19. Tarvainen TVJ, Yu W, Gonzalez J. Development of MorphHand: design of an underactuated anthropomorphic rubber finger for a prosthetic hand using compliant joints. In: Proceedings of the 2012 IEEE International Conference on Robotics and Biomimetics. 2012:142-147.

20. Morita T, Sugano S. Design and development of a new robot joint using a mechanical impedance adjuster. In: Proceedings of IEEE International Conference on Robotics and Automation. 1995:2469-2475.
21. Plettenburg DH. Electric versus pneumatic power in hand prostheses for children. J Med Eng Technol. 1989;13:124-128.

22. Plettenburg DH. Pneumatic actuators: a comparison of energy-to-mass ratios. In: Proceedings of the 2005 IEEE 9th International Conference on Rehabilitation Robotics. 2005:545-549.

23. SQUSE Inc. [PM-10P]. Available from: http://www.squse.co.jp/ product/detail.php?id=9. Accessed April 17, 2015. Japanese.

24. Nidec-Shimpo Corporation. FGP-50. Available from: http://www.nidecshimpokeisoku.jp/en/products/01/. Accessed April 17, 2015.

25. Futaba Corporation. RS405CB. Available from: http://www.futaba. co.jp/en/robot/5350/RS40x.html. Accessed April 17, 2015.

26. Sekine M, Tsuchiya N, Kita K, et al. Designing and testing a hybrid lightweight shoulder prosthesis. In: Proceedings of the 36th Annual International Conference of the IEEE Engineering in Medicine and Biology Society. 2014:2500-2503.

27. Sekine M, Fernandez-Vargas J, Hayata N, et al. A hybrid lightweight shoulder prosthesis with an antagonistic mechanism by PEAs. In: Proceedings of the 2014 International Workshop on Wearable Robotics. 2014;39.

28. Wen JT, Murphy S. Stability analysis of position and force control for robot arms. IEEE Trans Automat Contr. 1991;36:365-370.
Medical Devices: Evidence and Research

\section{Publish your work in this journal}

Medical Devices: Evidence and Research is an international, peerreviewed, open access journal that focuses on the evidence, technology, research, and expert opinion supporting the use and application of medical devices in the diagnosis, treatment and management of clinical conditions and physiological processes. The identification of novel

\section{Dovepress}

devices and optimal use of existing devices which will lead to improved clinical outcomes and more effective patient management and safety is a key feature. The manuscript management system is completely online and includes a quick and fair peer-review system. Visit http://www. dovepress.com/testimonials.php to read real quotes from authors. 